\title{
Thigh muscle size and vascular function after blood flow- restricted elastic band training in older women
}

\author{
Tomohiro Yasuda ${ }^{1,2,3}$, Kazuya Fukumura ${ }^{1}$, Takanobu Tomaru ${ }^{3}$ and Toshiaki \\ Nakajima ${ }^{1,4}$ \\ ${ }^{1}$ Graduate School of Medicine, University of Tokyo, Tokyo, Japan \\ ${ }^{2}$ School of Nursing, Seirei Christopher University, Shizuoka, Japan \\ ${ }^{3}$ Faculty of Medicine, Toho University, Chiba, Japan \\ ${ }^{4}$ Heart Center, Dokkyo Medical University Hospital, Tochigi, Japan \\ Correspondence to: Tomohiro Yasuda, email: tomohiro-y@seirei.ac.jp \\ Keywords: sarcopenia, vascular occlusion, muscle hypertrophy, resistance exercise, arterial stiffness, Gerotarget \\ Received: December 08, 2015 Accepted: May 12, $2016 \quad$ Published: May 23, 2016
}

\section{ABSTRACT}

We examined the effect of elastic band training with blood flow restriction (BFR) on thigh muscle size and vascular function in older women. Older women were divided into three groups: low-intensity elastic band BFR training (BFR-Tr, $n=10)$, middleto high-intensity elastic band training ( $M H-T r, n=10)$, and no training (Ctrl, $n=10)$ groups. BFR-Tr and MH-Tr groups performed squat and knee extension exercises using elastic band, 2 days/week for 12 weeks. During BFR-Tr exercise session, subjects wore pressure cuffs around the most proximal region of both thighs. The following measurements were taken before (pre) and 3-5 days after (post) the final training session: MRI-measured muscle cross-sectional area (CSA) at mid-thigh, maximum voluntary isometric contraction (MVIC) of knee extension, central systolic blood pressure (c-SBP), central-augmentation index (C-AIx), cardio-ankle vascular index testing (CAVI), ankle-brachial pressure index (ABI). Quadriceps muscle CSA (6.9\%) and knee extension MVIC $(13.7 \%)$ were increased $(p<0.05)$ in the BFR-Tr group, but not in the MH-Tr and the Ctrl groups. Regarding C-SBP, C-AIX, CAVI and ABI, there were no changes between pre- and post- results among the three groups. Elastic band BFR training increases thigh muscle CSA as well as maximal muscle strength, but does not decrease vascular function in older women.

\section{INTRODUCTION}

Skeletal muscle atrophy with aging (sarcopenia) inhibits mobility and increases the risk of falls, fractures, disability, and heart disease [1, 2]. High-intensity resistance training $(\mathrm{HI}-\mathrm{Tr}, \geq 70 \%$ 1-repetition maximum: 1RM) improves skeletal muscle morphology and function in young and older adults [3]. Since HI-Tr also improve insulin resistance and type-2 diabetes in the elderly [4, $5]$, the HI-Tr prevents and improves the sarcopenia in the elderly [6]. In general, traditional resistance exercise required for weight machines/free weights, but this method is not be practical and may even be dangerous. Thus, the effectiveness of alternative exercise methods should be investigated.

Elastic bands/tubing have been used practically (portable, less expensive, easy to used) in rehabilitative medicine and in health enhancement for resistance training [7, 8]. Additionally, home-based resistance-training program for older adults using elastic bands improves muscle strength [9]. However, since elastic resistance training is commonly performed using a low-to-moderate resistance level, this training typically has little or no effect on muscle hypertrophy $[9,10]$.

In the past 15 years, several studies revealed that muscle hypertrophy can be produced with lowintensity resistance training $(\sim 30 \% 1 \mathrm{RM})$ with blood flow restriction (BFR-Tr) regardless of age $[11,12]$. This 
Table 1: Changes in anthropometric variables after 12 weeks of training period.

\begin{tabular}{|c|c|c|c|c|c|c|c|c|c|}
\hline & \multicolumn{3}{|l|}{ BFR-Tr } & \multicolumn{3}{|l|}{ MH-Tr } & \multicolumn{3}{|l|}{ Ctrl } \\
\hline & Pre & Post & $\%$ & Pre & Post & $\%$ & Pre & Post & $\%$ \\
\hline \multicolumn{10}{|c|}{ Anthropometric variables } \\
\hline Age, yrs & $70(6)$ & & & $72(7)$ & & & $68(6)$ & & \\
\hline Standing height, $\mathrm{m}$ & $1.53(0.06)$ & & & $1.52(0.06)$ & & & $1.57(0.07)$ & & \\
\hline Body mass, $\mathrm{kg}$ & $48.3(5.8)$ & $48.4(5.9)$ & 0.0 & $48.7(8.1)$ & $48.3(7.2)$ & -0.7 & $55.0(7.1)$ & $54.8(6.9)$ & -0.4 \\
\hline $\mathrm{BMI}, \mathrm{kg} / \mathrm{m}^{2}$ & $20.8(2.5)$ & $20.7(1.9)$ & 0.2 & $20.9(2.1)$ & $20.7(1.8)$ & -0.7 & $22.3(2.8)$ & $22.2(2.7)$ & -0.4 \\
\hline Mid-thigh girth, cm & $43.9(3.8)$ & $44.2(3.7)$ & 0.7 & $45.1(4.0)$ & $44.9(4.1)$ & -0.4 & $46.9(2.8)$ & $46.4(3.1)$ & -1.1 \\
\hline Lower leg girth, $\mathrm{cm}$ & $32.8(2.3)$ & $32.9(2.1)$ & 0.3 & $32.6(3.3)$ & $32.8(3.2)$ & 0.6 & $34.4(1.8)$ & $34.2(1.9)$ & -0.4 \\
\hline
\end{tabular}

Notes: Data are given as mean $( \pm \mathrm{SD})$. BFR-Tr $=$ low-intensity elastic band BFR training; $\mathrm{MH}-\mathrm{Tr}=$ middle-to-high-intensity elastic band training; Ctrl $=$ non-BFR resistance training; BMI = body mass index; Mid-thigh girth $=$ at $50 \%$ thigh length; Lower leg girth $=$ at $30 \%$ lower leg length.

suggests that BFR-Tr using elastic bands for resistance may be an effective home-based resistance training program for promoting both muscle hypertrophy and strength in older adults. Unfortunately, low-intensity elastic band BFR training-induced muscle hypertrophy gain are not fully understood.

In general, prevention and treatment for decreased arterial compliance or stiffness is important $[13,14]$. Previous studies are split on whether HI-Tr decrease $[15,16]$ arterial compliance in young and older adults or not $[17,18]$. This means that the HI-Tr has a potentially decrease regarding arterial function in older adults. On the other hand, BFR-Tr could improve or maintain arterial function in young and older adults [19-21]. Therefore, we hypothesized that BFR-Tr produces muscle hypertrophy with a low risk of increased arterial stiffness in older adults.

Sarcopenia is muscle specific and that greater quadriceps muscle loss was found in older adults [22,23]. Thus, the purpose of this study was to examine the effect of low-intensity elastic band training with BFR on thigh muscle size and arterial stiffness in older adults.

\section{RESULTS}

Before training, there were no significant differences among three groups for age $(p=0.383)$ and anthropometric variables (standing height, $p=0.125$; body mass, $p=$ 0.783; BMI, $p=0.311$; mid-thigh girth, $p=0.195$; lower leg girth, $p=0.257$ ), MVIC (knee extension, $p=0.229$; knee flexion, $p=0.363$ ), muscle CSA (quadriceps, $p=$ 0.122 ; adductors, $p=0.218$; hamstrings, $p=0.148$; gluteus maximus, $p=0.540$ ) (Table 1$)$, hemodynamic parameter and vascular function ( $p=0.458-0.941)$, coagulation system and creatine kinase $(p=0.251-0.321)$ except for leg press $1 \mathrm{RM}$ strength $(p=0.044)$ and D-dimer $(p=$ 0.029 ) (Table 2). A significant group-by-time interaction was not observed for body weight $(p=0.655)$ and BMI $(p=0.928)$ in three groups following the training period.

\section{Acute effect of BFR-Tr and MH-Tr}

A significant group by time interaction was observed for anterior mid-thigh $(p=0.032)$ but not for posterior mid-thigh MTH $(p=0.731)$. Immediately after the exercise session, mean MTH was increased in both BFR-Tr and MH-Tr groups $(p=0.001$ and $p=0.002$, respectively), and the percent change in anterior mid-thigh MTH tended to be greater $(p=0.062)$ in the BFR-Tr group (pre: $3.8(0.6) \mathrm{cm} v s$. post: $4.2(0.6) \mathrm{cm}$ : 10.6\%) compared with MH-Tr group (pre: $3.7(0.5) \mathrm{cm} v s$. post: $3.9(0.6)$ $\mathrm{cm}$ : 6.3\%) (Figure 1).

There were no differences between BFR-Tr and $\mathrm{MH}-\mathrm{Tr}$ groups in the elastic band elongation ( $p=0.734$ for squatting and $p=0.738$ for knee extension, respectively) (Table 3), ratings of perceived exertion ( $p=0.075$ for squatting and $p=0.341$ for knee extension, respectively), and range of motion ( $p=0.073$ (knee joint) and $p=0.311$ (hip joint) for squatting and $p=0.387$ for knee extension, respectively). Heart rate was higher in the BFR-Tr group than in the MH-Tr group for squatting $(p=0.515)$, but not for knee extension ( $p=0.047$ ) (Table 4).

\section{Mid-thigh anterior $\mathbf{5 0 \%}$}

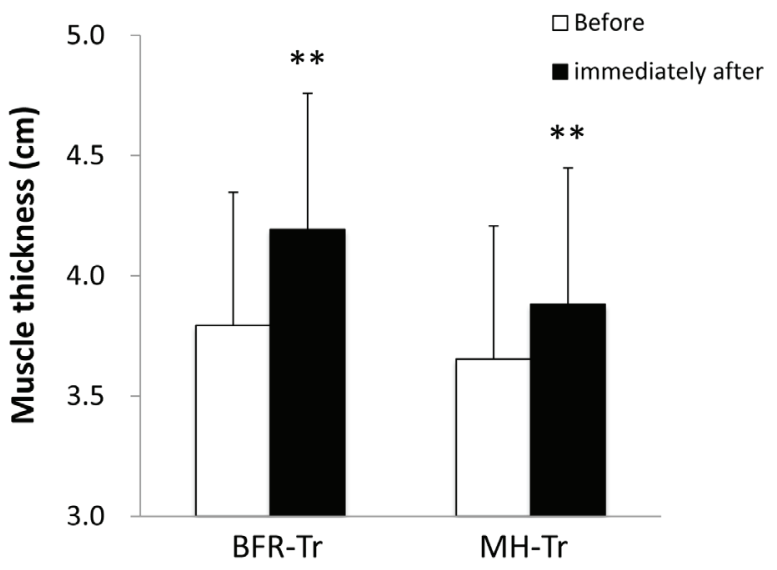

Figure 1: Muscle thickness of mid-thigh at anterior $50 \%$ before and immediately after exercise session. Data are given as mean $( \pm \mathrm{SD})$. **Different from before, $P<$ 0.01 . 
Table 2: Changes in hemodynamic parameter and vascular function, coagulation system and muscle damage after 12 weeks of training period.

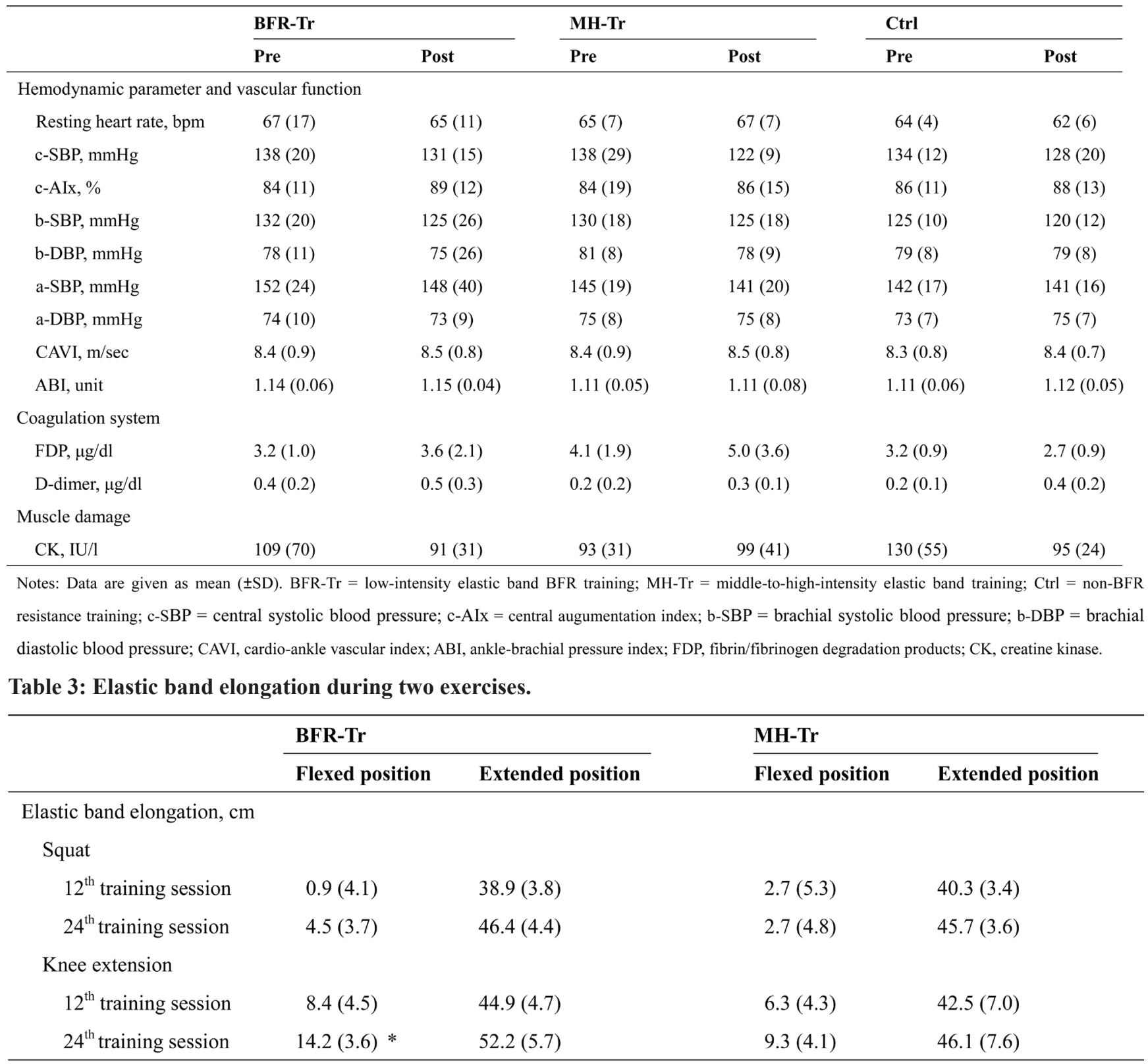

Notes: Data are given as mean $( \pm \mathrm{SD}) . \mathrm{BFR}-\mathrm{Tr}=$ low-intensity elastic band BFR training; $\mathrm{MH}-\mathrm{Tr}=$ middle-to-high-intensity elastic band training. $* \mathrm{p}<0.05$, BFR-Tr versus MH-Tr.

Figure 2: Muscle cross-sectional area (CSA) of quadriceps pre- and post-training period. Data are given as mean $( \pm \mathrm{SD}) . * *$ Different from pretraining, $P<0.01$.

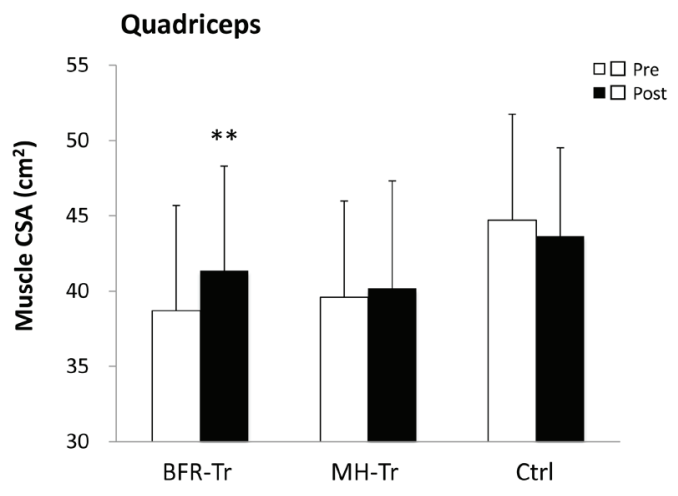


Table 4: Ratings perceived exertion, heart rate and range of motion during two exercises.

BFR-Tr

Mean ( \pm SD)
MH-Tr

Mean ( \pm SD) $\quad$ Range

Ratings of perceived exertion, OMNI-Res Scale

Squat
$12^{\text {th }}$ training session
$7.7(1.4) *$
6-10
$5.8(1.5)$
5-7
$24^{\text {th }}$ training session
$7.2(1.4)$
6-10
$6.4(1.0)$
6-8
Knee extension

$$
\begin{aligned}
& 12^{\text {th }} \text { training session } \\
& 24^{\text {th }} \text { training session }
\end{aligned}
$$
$8.2(1.4) *$
6-10
$7.0(0.9)$
6-8
$8.0(1.4)$
$6-10$
$7.5(1.3)$
6-9

Heart rate, bpm

Squat

$12^{\text {th }}$ training session

$129(21)$

101-167

119 (19)

$82-150$

$24^{\text {th }}$ training session

129 (22)

96-164

129 (16)

107-155

Knee extension

$$
\begin{aligned}
& 12^{\text {th }} \text { training session } \\
& 24^{\text {th }} \text { training session }
\end{aligned}
$$

93-167

108 (19)

$87-141$

123 (20)

94-166

111 (12)

92-128

Range of motion, degree

Squat

Hip joint

$\begin{array}{llccc}12^{\text {th }} \text { training session } & 69(8) & 60-81 & 67(16) & 41-92 \\ 24^{\text {th }} \text { training session } & 66(11) & 52-86 & 59(11) & 45-81 \\ \text { Knee joint } & & & \\ 12^{\text {th }} \text { training session } & 81(15) & 61-101 & 96(22) & 62-129 \\ 24^{\text {th }} \text { training session } & 93(16) & 73-126 & 106(17) & 75-134\end{array}$

Knee extension

Knee joint

$12^{\text {th }}$ training session

$80(6)$

$75-90$

84 (6)

73-94

$24^{\text {th }}$ training session

85 (5)

76-95

$85(12)$

70-104

Notes: Data are given as mean $( \pm \mathrm{SD})$. BFR-Tr $=$ low-intensity elastic band BFR training; MH-Tr $=$ middle-to-high-intensity elastic band training. ${ }^{*} \mathrm{p}<0.05$, BFR-Tr versus MH-Tr.

\section{Chronic effect of BFR-Tr and MH-Tr}

A significant group by time interaction was observed for muscle CSA ( $p<0.001$ for quadriceps), MVIC ( $p=$
0.028 for knee extension) and leg press 1RM $(p<0.001)$, but not for knee extension 1RM $(p=0.076)$. Quadriceps muscle CSA $(6.9 \%)$ was increased $(p<0.001)$ in the BFRTr group, but not in the MH-Tr $(1.5 \%, p=0.871)$ and the Ctrl $(-2.2 \%, p=0.395)$ groups (Figure 2). Knee extension 
Table 5: Effect size in muscle size and strength after 12 week of training period.

\begin{tabular}{|c|c|c|c|}
\hline & BFR-Tr & MH-Tr & Ctrl \\
\hline & Pre to post & Pre to post & Pre to post \\
\hline \multicolumn{4}{|l|}{ Muscle CSA } \\
\hline Quadriceps & $0.38^{a}$ & 0.09 & -0.15 \\
\hline Adductors & -0.05 & 0.10 & -0.23 \\
\hline Hamstrings & 0.03 & -0.02 & -0.25 \\
\hline Gluteus maximus & 0.14 & 0.11 & -0.01 \\
\hline \multicolumn{4}{|l|}{ MVIC } \\
\hline Knee extension & $0.64^{b}$ & $0.27^{\mathrm{a}}$ & $0.29^{\mathrm{a}}$ \\
\hline Knee flexion & 0.00 & 0.03 & -0.24 \\
\hline \multicolumn{4}{|l|}{$1 \mathrm{RM}$} \\
\hline Knee extension & $0.29^{\mathrm{a}}$ & 0.11 & 0.05 \\
\hline Leg press & $0.74^{\mathrm{b}}$ & $0.79^{b}$ & 0.10 \\
\hline
\end{tabular}

Notes: Data are given as mean $( \pm \mathrm{SD})$. BFR-Tr $=$ low-intensity elastic band BFR training; $\mathrm{MH}-\mathrm{Tr}=$ middle-to-high-intensity elastic band training; $\mathrm{Ctrl}=$ non-BFR resistance training; $\mathrm{CSA}=$ cross-sectional area; MVIC $=$ maximal voluntary isometric contraction; $1 \mathrm{RM}=$ one-repetition maximum. ${ }^{\mathrm{a}}=$ small effect size ${ }^{\mathrm{b}}=$ moderate effect size $;^{\mathrm{c}}=$ large effect size.

MVIC (13.7\%) was increased $(p=0.028)$ in the BFR-RT group, but not in the MH-Tr $(p=0.196)$ or $\operatorname{Ctrl}(p=0.810)$ groups (Figure 3a). Knee extension 1RM (7.6\%) tended to be increased $(p=0.076)$ in the BFR-RT group, but not in the $\mathrm{MH}-\operatorname{Tr}(p=0.605)$ or $\mathrm{Ctrl}(p=0.998)$ groups (Figure $3 b)$. Leg press $1 \mathrm{RM}(16.4 \%$ and $17.6 \%)$ was increased (both $p<0.001$ ) in the BFR-RT and the MH-Tr groups, respectively, but not in the $\mathrm{Ctrl}(p=0.912)$ group (Figure $3 c)$. The magnitude of effect size between pre- and posttraining was larger in the BFR-Tr group than in the MHTr group for quadriceps muscle CSA (small vs. trivial), knee extension MVIC (moderate vs. small), and knee extension 1RM (small vs. trivial), but not for leg press 1RM (moderate $v s$. moderate) (Table 5).

No significant group-by-time interaction was observed for muscle CSA ( $p=0.104$ for adductors, $p=$ 0.119 for hamstrings, and $p=0.623$ for gluteus maximus), MVIC ( $p=0.419$ for knee flexors), hemodynamic parameter and vascular function $(p=0.474-0.929)$, coagulation system $(p=0.599-0.753)$, and creatine kinase $(p=0.099)$ (Table 2).

\section{DISCUSSION}

BFR-Tr using weight machines/free weight leads to increased thigh muscle size and the maintenance of arterial stiffness $[20,21]$. In this study, low-load, elastic band resistance training (squatting and knee extension) with BFR can leads to increase in quadriceps muscle CSA as well as maximal strength in older women. In addition, the hemodynamic parameter, vascular function, coagulation system and creatine kinase are no changes.

In this study, MH-Tr group did not increase muscle hypertrophy for lower limb. Previous study [24] reported that elastic band $\mathrm{MH}-\mathrm{Tr}$ is not easy to induce the muscle hypertrophy even for upper limbs. Since resistance level by elastic band is required greater in lower limb compared with upper limb, it is increasingly difficult to control in direction for resistance, ROM for active muscles, and fixation for handgrip. On the other hand, the BFR-Tr group increased in quadriceps muscle CSA. Additionally, the observed gain in quadriceps muscle CSA $(0.29 \%$ per session) provided comparable results as BFR training using free weights $(0.33 \%$ per session) [21]. Thus, lowintensity elastic band training with BFR can provide a sufficient hypertrophic stimulus for thigh muscles in older adults.

There are some trigger mechanisms underlying the BFR-Tr-induced muscle hypertrophy. In HI-Tr, myogenic stem cells most likely activate muscle protein synthesis [25]. BFR-Tr leads to proliferation of myogenic stem 
(a) Knee extension

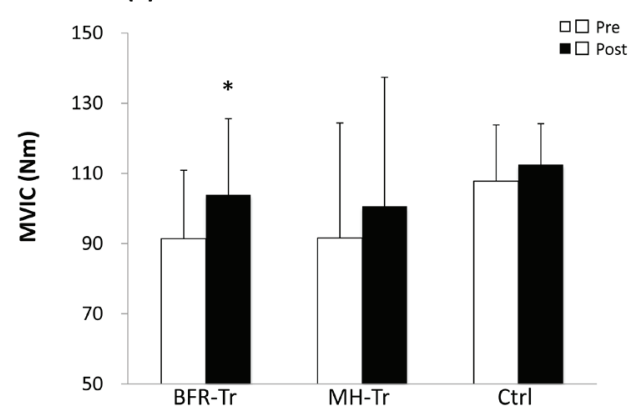

(b) Knee extension

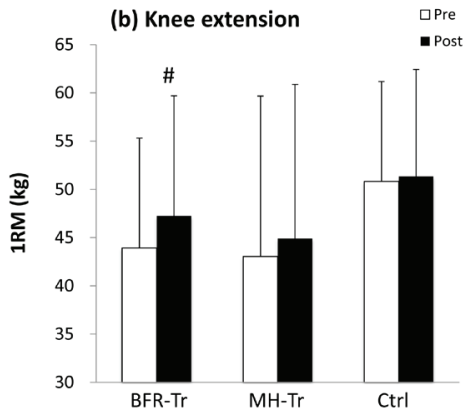

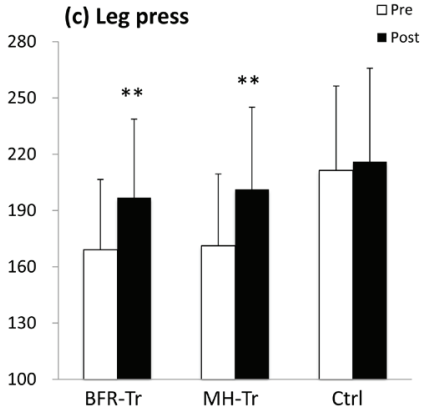

Figure 3: Maximum voluntary isometric contraction (MVIC) of knee extension. a. and $1 \mathrm{RM}$ of knee extension b. and leg press c. pre- and post- training period. ${ }^{*}$ Different from pretraining, $P<0.01$. ${ }^{*}$ Different from pretraining $P<0.05,{ }^{*}$ Different from pretraining, $P=0.08$.

(a) Squat

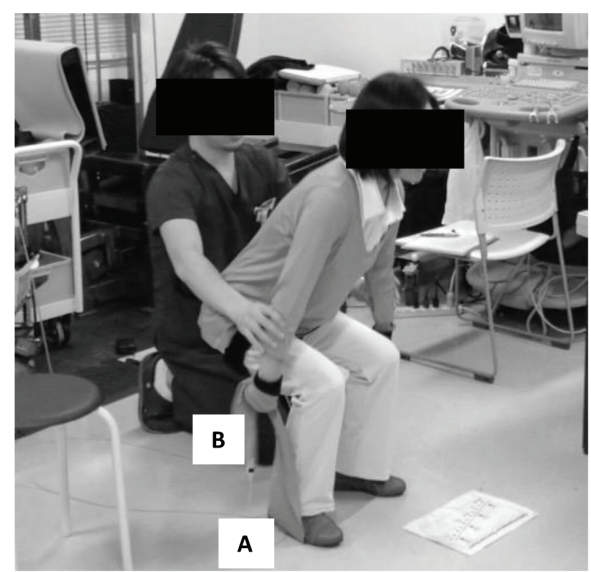

(b) Knee extension

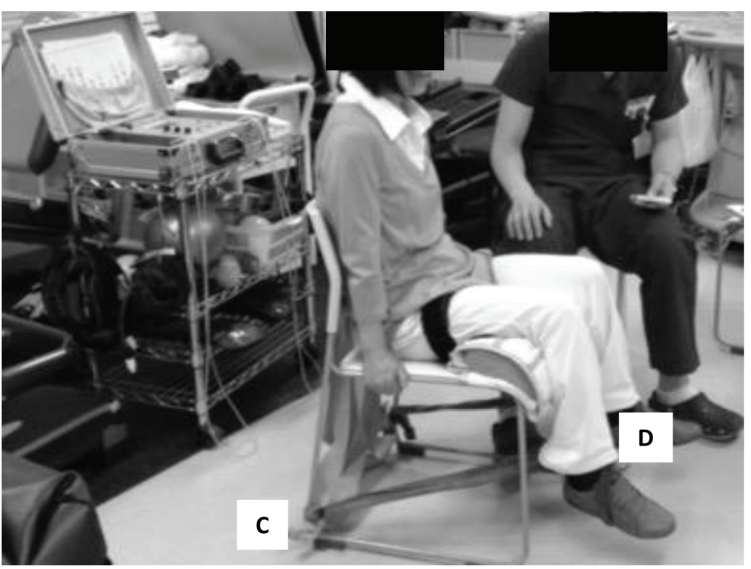

Figure 4: Illustrations show the body position used for two exercises. Squat a. and knee extension b. exercises were performed with an elastic band. $\mathrm{A}=$ outside foot; $\mathrm{B}=$ grip; $\mathrm{C}=$ tie on posterior chair leg; $\mathrm{D}=$ ankle.

\section{Training period}

Pre

\begin{tabular}{|l|l|l|l|l|l|l|l|l|l|l|l|}
\hline $1 w k$ & $2 w k$ & $3 w k$ & $4 w k$ & $5 w k$ & $6 w k$ & $7 w k$ & $8 w k$ & $9 w k$ & $10 w k$ & $11 w k$ & $12 w k$ \\
\hline
\end{tabular}

$\begin{array}{ll}\text { Muscle CSA } & \uparrow \\ \text { MVIC } & \uparrow \\ \text { 1RM } & \uparrow \\ \text { Vascular function } & \uparrow \\ \text { Blood samples } & \uparrow\end{array}$

RPE

Heart rate

MTH

ROM

Elastic band elongation

$$
\begin{aligned}
& \uparrow \uparrow \uparrow \uparrow \uparrow \uparrow \uparrow \uparrow \uparrow \uparrow \uparrow \uparrow \uparrow \uparrow \uparrow \uparrow \uparrow \uparrow \uparrow \uparrow \uparrow \uparrow \uparrow \uparrow \\
& \uparrow \uparrow \uparrow \uparrow \uparrow \uparrow \uparrow \uparrow \uparrow \uparrow \uparrow \uparrow \uparrow \uparrow \uparrow \uparrow \uparrow \uparrow \uparrow \uparrow \uparrow \uparrow \uparrow \uparrow \\
& \uparrow \\
& \uparrow \\
& \uparrow \\
& \uparrow \\
& \uparrow
\end{aligned}
$$

Figure 5: Experimental timeline. $\mathrm{CSA}=$ cross-sectional area; $\mathrm{MVIC}=$ Maximum voluntary isometric contraction; $1 \mathrm{RM}=$ one repetition maximum; $\mathrm{RPE}=$ ratings of perceived exertion; $\mathrm{MTH}=$ muscle thickness; $\mathrm{ROM}=$ range of motion. 
cells, an increase of myonuclei in skeletal muscle, and marked myofiber hypertrophy [26]. Therefore, myogenic stem cell-derived myonuclei may provide an improved capacity for myofibrillar gene transcription, which enhanced activity of muscle protein synthesis for BFR-Tr by using elastic bands as well as weight machines and/or free weights.

BFR-Tr-induced muscle cell swelling may activates to the anabolic benefits of BFR [27, 28, 29]. With respect to effect size for acute MTH, an index of muscle cell swelling, of the anterior thigh, the BFR-Tr group showed a greater MTH response compared with the $\mathrm{MH}-\mathrm{Tr}$ group (0.72 vs. 0.41). It is known that acute cell swelling simulates anabolic processes through an increase in protein synthesis and a decrease in proteolysis [30, 31]. Thus, the BFR exercise-induced enhancement of muscle protein metabolism may increase in quadriceps muscle size.

In the BFR-Tr group, increased muscle strength (\%change per session and effect size) in this study was lower than that in the previous study [21] for knee extension ( $0.32 \%$ vs. $1.09 \%$ and 0.29 vs. 0.70$)$ and leg press $1 \mathrm{RM}(0.68 \%$ vs. $1.39 \%$ and 0.74 vs. 0.98$)$. In general, elastic band training did not large stimulation in the 1RM strength because resistance type is dramatic difference between elastic bands and weight machines. Thus, it appears that the difference in improvement in muscle strength between elastic bands and weight machines is attributed to unequal neural adaptations.

An elastic band resistance training is well tolerated, as indicated by non-exacerbation of chronic disease conditions and lack of training-induced injury [8,9]. In this study, the ratings of perceived exertion were higher in the BFR-Tr group compared with the MH-Tr group, but the hemodynamic parameter, vascular function, coagulation system and creatine kinase were not changed for the both groups. In particular, central blood pressure and cardiac afterload were not increased by BFR-Tr group as well as MH-Tr group. In general, endothelial function, as assessed by brachial flow-mediated dilatation (FMD), has an inverse relationship with central arterial stiffness [32]. FMD was not assessed in this study, but previous study reported that FMD was not decreased by 12 weeks of BFR-Tr [21]. This may explain why there was not an increase in central blood pressure and cardiac afterload following BFR-Tr. Together, BFR-Tr using elastic bands is a relatively safe training method. However, it should be noted that the possibility of side effects cannot be denied when subjects perform such training until near exhaustion or particularly to complete exhaustion $[33,34]$.

The present study has some limitations. First, it should be noted that our sample size was small. In this study, the effect size in muscle CSA (quadriceps: 0.38 for BFR-Tr), MVIC (knee extension: 0.64 for BFR-Tr and 0.27 for $\mathrm{MH}-\mathrm{Tr}$ ) and 1RM (knee extension: 0.29 for BFR-Tr, leg press: 0.74 for BFR-Tr and 0.79 for $\mathrm{MH}-$
Tr) following the training period was not high (low-tomoderate level of effect size). Totally, the effects of elastic bands BFR-Tr on muscle size and strength was small compared with weight machines BFR-Tr, although the training protocol was basically similar between the two studies (this study $v s$. previous study [21]). Second, it was very difficult to evaluate the exercise intensity using elastic bands. As in the case of the previous study [24], we employed a similar method by adjusting the distance of the band stretch based on OMNI-Res scale [35], but the precise exercise intensity was merely estimated. Third, the type of band used in our study presented close to the same resistance level (gold, black, etc.) for each group, consequently the arbitrary pressure was used. Forth, since post-training measurements were performed 3-7 days, the difference of measurement date might suppress the results. Lastly, because our participants were only old women, it is uncertain whether the results pertain to old men. Additional research into these issues is needed.

In conclusion, low-intensity squatting and knee extension training with BFR using elastic bands for resistance elicited marked gains in quadriceps muscle CSA and strength, and did not decrease vascular function in older women. Thus, our results demonstrate that lowintensity, elastic band BFR training would be beneficial in the development of safe and effective methods of sarcopenia care and prevention in older adults.

\section{MATERIALS AND METHODS}

\section{Participants}

Thirty women (aged 61-86 years) volunteered to participate in the study and were selected according to the exclusion criteria (blood pressure $>160 / 100$ $\mathrm{mmHg}$, body mass index $>30 \mathrm{~kg} / \mathrm{m}^{2}$, history of anemia, cerebrovascular disease, myocardial infarction and arthroscopic joint surgery) used to define "medically stable" older participants for exercise studies proposed by Greig et al. [36]. In addition, volunteers who suffered from a chronic disease such as severe hypertension $(>170 / 110$ $\mathrm{mmHg}$ ), orthopedic disorders, deep venous thrombosis, peripheral vascular disease, or cognitive dysfunction were excluded from the study. The participants in this study were physically active, with nine (BFR-Tr, $n=3$; MH-Tr, $n=4$; Ctrl, $n=2$ ) of 30 participated in regular aerobictype exercise (walking, jogging or cycling; 2-3 times per week for approximately $30 \mathrm{~min}$ in duration). None of the participants had participated in resistance-type training for a minimum of 6 months prior to the study. All participants were non-smokers, were free of overt chronic disease as assessed by medical history, physical examination, and complete chemistry and hematologic evaluation. No participant was on hormonal replacement therapy. They 
were randomly divided into a low-intensity elastic band BFR training (BFR-Tr, $\mathrm{n}=10$, mean \pm SD: $70 \pm 6$ years) or a middle-to-high-intensity elastic band training (MH-Tr, $\mathrm{n}=10,72 \pm 7$ years $)$ or no training $(\mathrm{Ctrl}, \mathrm{n}=10,68 \pm 6$ years) groups. Eight (BFR-Tr, $\mathrm{n}=3$; $\mathrm{MH}-\mathrm{Tr}, \mathrm{n}=3$; Ctrl, $\mathrm{n}=2$ ) of all participants were classified as "hypertension (>140/90 mmHg)" [37], but nobody took medicine for high blood pressure. All participants were informed of the risks associated with involvement in the study and signed an informed consent document before participation. The principles of the World Medical Association Declaration of Helsinki and the American College of Sports Medicine Guidelines for Use of Human Subjects were adopted in this study. The study was approved by the Ethics Committee of the University of Tokyo.

\section{Training protocol}

Two training groups performed bilateral squat and knee extension exercise training 2 days/week for 12 weeks (Figure 4). The MH-Tr group exercised at two exercise intensities ranging from 5.6 to 8.4 on the OMNI perceived exertion scale for resistance exercise (OMNI-RES) for active muscle scale ( 0 -extremely easy to 10 -extremely hard) which has been noted to correspond to exercise intensity levels ranging from approximately $70 \%$ to $90 \%$ of $1 \mathrm{RM}$ for women [35]. If the intensity range was below 5 or above 9 , the intensity of the two exercises was modified to either increase or decrease the resting length of the elastic band. As a result of the pilot study, the MHTr groups used two gold (Max) bands for squatting, and one gold band for knee extension (Hygenic Corporation, Akron, Ohio, USA).

The BFR-Tr group used one gold band for squatting, and one black (Special Heavy) band for knee extension. The gold band was approximately twice the resistance level as the black band [38], thus the two exercises for the BFR-Tr group were one-half (low-intensity level) the intensity as that for the $\mathrm{MH}-\mathrm{Tr}$ group. These training exercises were performed under the close supervision of those with technical knowledge in BFR training. The BFR-Tr group exercised at two exercise intensities ranging from 5 to 9 on the OMNI Resistance for active muscle scale. If the intensity range was below 5 or above 9 , the intensity of the two exercises was modified to either increase or decrease the resting length of the elastic band and/or restriction pressure intensity. In both groups, the repetition duration was 2.6 seconds (1.3-second concentric and 1.3-second eccentric exercise cycle) and 2.0 seconds (1.0-second concentric and 1.0-second eccentric exercise cycle) for squat and knee extension, respectively.

One week before the start of the training study, both groups performed practice sessions for the maximum voluntary isometric contraction (MVIC) and knee extension and leg press 1RM test. In addition, the BFRTr participants became familiar with the BFR stimulus.
Three or four days before training, the MVIC and 1RM were determined. In the BFR-Tr group, training was set at 75 repetitions $(30,15,15$, and 15 repetitions, with a 30 -second resting period between sets) for both exercises (90-second rest between exercises). This protocol is consistent with submaximal BFR studies [12,20,21,39]. Once the pneumatic cuffs were inflated, they remained inflated for the two exercises, including during the resting periods between sets and exercises. On the other hand, in the $\mathrm{MH}-\mathrm{Tr}$ group, training was set at 37 or 38 repetitions $\left(13,13\right.$ (at $^{\text {st }}-12^{\text {th }}$ training session) or 12 (at $13^{\text {th }}-24^{\text {th }}$ training session), and 12 repetitions with 30 -second rests between sets) for both exercises ( 90 -second rests between exercises). Exercise repetition for BFR-Tr group was twice that for $\mathrm{MH}-\mathrm{Tr}$ group, although the exercise intensities for $\mathrm{MH}-\mathrm{Tr}$ group were twice that for BFR-Tr group. Therefore, the same training volume (exercise intensity $\mathrm{X}$ repetitions) was set between the two training groups.

During squat exercises, knee joint range of motion (ROM) and hip joint ROM were approximately 100-0 degrees and 100-0 degrees, respectively, while during knee extension exercises, knee joint ROM was approximately 95- $15^{\circ}$ and hip joint ROM was maintained at $90^{\circ}$ (with $180^{\circ}$ being full extension). Subjects were instructed not to let the band snap them back to the start position but rather to consciously control the return movement such that it would take twice as long as the stretching movement. In addition, elastic band elongation was measured by tape measure for squat (distance from outside foot [a] to grip [b]) and knee extension (distance from tie on posterior chair leg [c] to ankle [d]) exercises (Figure 4). The measurements of ROM and elastic band elongation were completed at the $6^{\text {th }}$ week and $12^{\text {th }}$ week of the training period (Figure 5).

\section{Blood flow restriction}

During the training sessions, BFR-Tr participants wore a specially designed pneumatic cuff (50 $\mathrm{mm}$ width, KAATSU Master, KAATSU Japan Co., Ltd., Tokyo, Japan) around the most proximal portion of both thighs. On the first day of training, the cuffs were set at $50 \mathrm{mmHg}$ and air pressure was gradually inflated to $120 \mathrm{mmHg}$ (Day 1). The air pressure was increased by $10-20 \mathrm{mmHg}$ at each subsequent training session until a pressure of approximately $200 \mathrm{mmHg}$ was reached if the subject could perform at a high level of pressure intensity. The restriction pressure was selected in accordance with a previous study [40]. The mean pressure intensity throughout the period of training was $161 \pm 12 \mathrm{mmHg}\left(160-200 \mathrm{mmHg}\right.$ at $24^{\text {th }}$ training session). Immediately after the two exercises, the pressure cuff was quickly removed. The amount of time under blood flow restriction was approximately 10-11 min. 


\section{Measurements schedule}

Subject testing took place before the start of the study (pre) and 3-7 days after (post) the 12-week training period. The order of measurements were MRI, venous blood samples, vascular function tests [central systolic blood pressure (c-SBP), central-augmentation index (c-AIx), cardio-ankle vascular index testing (CAVI), ankle brachial pressure index (ABI)], maximum voluntary isometric contraction (MVIC) and 10RM strength. Figure 5 shows the testing schedule for each of the measurements taken during the 12 week experimental period. All data were obtained from the right side of the body. Considering the schedule of examiners, all subjects and variable technologists, the MRI (9:00 and 15:00 hours), venous blood samples and arterial function tests (after 6-7 hours of fasting, 9:00 and 15:00 hours), and MVIC (9:00 and 15:00 hours) measurements were obtained on two days. The subjects were instructed to not drink alcohol or caffeine during the 24-hour period and from performing any strenuous exercise during the 48 -hour period prior to pre- and post-training measurements.

\section{MRI-measured muscle CSA}

Muscle CSA was obtained using a MRI scanner (0.2-T Open MRI, Hitachi, Tokyo, Japan). A T-1 weighted, spin-echo, axial plane sequence was performed with a 500msec repetition time and a $23-\mathrm{msec}$ echo time. Subjects rested quietly in the magnet bore in a supine position with their legs extended. The top edge of the great trochanter was used as the origin point, and continuous transverse images with $10-\mathrm{mm}$ slice thickness ( 0 -mm interslice gap) were obtained from the top edge of the great trochanter to the lateral condyle of femur at pre- and post-training measurements. All MRI data were transferred to a personal computer for analysis using specially designed image analysis software (sliceOmatic, Tomovision Inc., Magog, QC, Canada). Skeletal muscle tissue cross-sectional area (CSA) data for the quadriceps, adductors and hamstrings at $50 \%$ of thigh length and for the gluteus maximus at the top edge of the great trochanter were digitized. The coefficient of variation of this measurement was less than $1.0 \%[21]$.

\section{Maximum voluntary isometric contraction}

MVIC of the knee extensors and flexors was determined by using a dynamometer (Biodex System 3, Sakai Medical Instrument, Tokyo, Japan). Participants were carefully instructed so that they became familiarized with the testing procedures of voluntary force production of the thigh muscles during several submaximal and maximal performances about 1 week before testing.
The participants were seated on a chair with their hip joint angle positioned at $85^{\circ}\left(0^{\circ}\right.$ at full extension). The center of rotation of the knee joint was visually aligned with the axis of the lever arm of the dynamometer and the ankle of the right leg was firmly attached to the lever arm of the dynamometer with a strap. Several warm-up contractions were performed before testing. Participants were then instructed to perform maximal isometric knee extension and flexion at a knee joint angle of $80^{\circ}$ and $40^{\circ}$ $\left(0^{\circ}\right.$ at full extension), respectively. If MVIC torque for the first two MVICs (60-sec rest interval) varied by more than $5 \%$, up to two additional MVICs were performed with a 60 -sec rest between trials. Participants were instructed to perform an MVIC as quickly as possible during a period of about 2 seconds. The highest MVIC value was used for data analysis. The test-retest reliability (ICC, SEM and minimal difference) was previously determined using the data of 9 older women measured twice within 7 days (at least one day apart) for MVIC $(0.917,5.03 \mathrm{Nm}, 13.9 \mathrm{Nm})$.

\section{Estimation of 1RM Strength}

One RM was estimated by the 10RM method (1-RM $=100 \cdot \mathrm{rep}$ mass $/(48.8+53.8 \cdot \exp [-0.075 \cdot \mathrm{reps}])[41]$ using a weight stack machine. Bilateral knee extension and leg press maximum dynamic strength (1RM) were assessed using an isotonic knee extension equipment (VR1, Cybex International, Inc.) and a leg press machine (Seated Leg Press, Life Fitness). After warming up, the testing load was set (approximately $80 \%$ of predicted 1RM). Each participant reached muscular failure for the load, and partial repetitions (where participants failed to lift through the entire ROM) did not count as RMs. If a participant had to perform a given repetition number for a given condition again, as a result of ease in obtaining the desired repetitions or failure to attain the repetition number, a 5-minute rest period was given and the condition was attempted again at an altered load. No participant had to perform a given repetition number test condition more than 3 times. Each participant performed the knee extension exercise, rested for 5 minutes, and then performed the leg press exercise. During estimated $1 \mathrm{RM}$ testing as well as training sessions, the parallel leg stance width was set at $100 \%$ of the shoulder-width for leg press exercises. The test-retest reliability (ICC, SEM and minimal difference) was previously determined using the data of 9 older women measured twice within 7 days (at least one day apart): $0.990,1.61 \mathrm{~kg}, 4.47 \mathrm{~kg}$ for knee extension 1RM and $0.990,3.44,9.53$ for leg press.

\section{Vascular function tests}

c-SBP and c-AIx, measurements were conducted in the seated position. The participants were instructed to fast 6-7 hours before testing and refrain from alcohol 
or caffeine intake for at least 12 hours prior to testing. The c-SBP was examined in the seated position in a quiet temperature-controlled room $\left(25-26^{\circ} \mathrm{C}\right)$. Radial artery pressure waveforms and brachial BP were recorded simultaneously using a fully automated device (HEM9000AI, Omron Healthcare Co., Ltd., Kyoto, Japan) to calculate late systolic pressure in the radial artery and to estimate c-SBP and c-AIx. There were significant positive correlations between aorta-SBP and radial-SBP $(r=0.95, p$ $<0.001)$ or aorta-AIx and radial-AIx $(r=0.91, p<0.001)$ [42]. The brachial BP was measured with an oscillometric manometer and the radial pulse waveforms were recorded noninvasively using an applanation tonometer, which consisted of a sensor unit with an array of 40 fine-pitch microtransducer elements and a monitor unit [43]. Then, CAVI and ABI were measured noninvasively using a VS1500 system (Fukuda Denshi Co., Ltd., Tokyo, Japan). The CAVI and ABI measurements were conducted in the supine position. Participants were asked to rest in a quiet temperature-controlled room $\left(25-26^{\circ} \mathrm{C}\right)$ for $20-30 \mathrm{~min}$. Electrocardiogram and heart sound were monitored. CAVI was automatically calculated using the formula; a $\{2 \rho /$ $\left.\Delta \mathrm{P} \times \operatorname{In}(\mathrm{Ps} / \mathrm{Pd}) \mathrm{PWV}^{2}\right\}+\mathrm{b}$ ( $\mathrm{a}$ and $\mathrm{b}$, constants; $\rho$, blood density; $\mathrm{P}$, difference in systolic and diastolic pressure; Ps, systolic pressure wave velocity; Pd, diastolic pressure; PWV, heart-ankle pulse wave velocity). The ankle-brachial pressure index (ABI) was calculated as the ratio of the systolic blood pressure in the ankle to the systolic blood pressure in the right arm [44]. To measure the stiffness the aorta, CAVI is essentially independent of blood pressure unlike PWV [44]. Therefore, arterial function was determined using CAVI. The test-retest reliability (ICC, SEM and minimal difference) was previously determined using the data of 9 older women measured twice within 7 days (at least one day apart): 0.974, $4.12 \mathrm{mmHg}, 11.4$ $\mathrm{mmHg}$ for c-SBP, $0.823,2.52 \%, 6.98 \%$ for c-AIx, 0.957 , $0.15 \mathrm{~m} / \mathrm{sec}, 0.42 \mathrm{~m} / \mathrm{sec}$ for CAVI and $0.439,0.04$ unit, 0.10 unit for ABI.

\section{Blood sampling and biochemical analyses}

Venous blood samples were obtained from the antecubital vein and measured for fibrin/fibrinogen degradation products (FDP), D-dimer and creatine kinase (CK). The plasma concentrations of these samples were measured at a commercial laboratory (SRL Inc., Tokyo, Japan) by following latex immunoassay (LIA) for FDP and D-dimer and spectrophotometry for NADPH formed by a hexokinase and D-glucose-6-phosphate-dehydrogenasecoupled enzymatic system for CK.

\section{Measurements of acute responses to training session}

\section{Ratings of perceived exertion}

During all training sessions, OMNI-RES based on a numerical scale of 0 to 10 were collected to assess subjective feelings of physical effort (i.e. exertion). OMNI-RES data were recorded immediately after the last set of each exercise [35].

\section{Ultrasound-measured muscle thickness}

Since muscle thickness (MTH) measurement using a B-mode ultrasound (Acuson Sequoia 512, Siemens, Tokyo, Japan) has the advantage of evaluating acute change in muscle cell swelling following exercises [29], the following measurements were made: MTH of the anterior and posterior mid-thigh, midway between the lateral condyle of the femur and greater trochanter; and posterior lower leg, at 30\% proximal between the lateral malleolus of the fibula and the lateral condyle of the tibia. Briefly, the measurements were carried out while the subjects stood with their elbows extended and relaxed. A $10.0 \mathrm{MHz}$ scanning head $(5.5 \mathrm{~cm}$ length probe) was placed on the skin perpendicular to the tissue interface. The scanning head was coated with a watersoluble transmission gel to provide acoustic contact without depressing the dermal surface. The subcutaneous adipose tissue-muscle interface and the muscle-bone interface were identified from the ultrasonic image. The perpendicular distance from the adipose tissue-muscle interface to the muscle-bone interface was considered to represent MTH. Ink markers on the anterior and posterior thigh and posterior lower leg were used to ensure similar positioning over repeated MTH measurements. The MTH was recorded before and immediately after the exercise bout. This measurement was completed at the $7^{\text {th }}$ week and $10^{\text {th }}$ week of the training period, and the average of two measurements for "before" or "immediately after" was represented as a single data point for statistical analysis, respectively. The test-retest reliability (ICC, SEM and minimal difference) was previously determined using the data of 9 older women measured twice within few days for MTH $(0.994,0.28 \mathrm{~cm}, 0.79 \mathrm{~cm})$.

\section{Heart rate}

During all training sessions, heart rate was recorded at baseline (pre) and immediately after the last set of each exercise (post) (Model 9560, Onyx II, Nonin Medical Inc., Plymouth, MN, USA). 


\section{Statistical analyses}

The results are expressed as mean \pm standard deviation (SD). The data were tested for normality using the Shapiro-Wilk test. Because all variables were normally distributed, parametric statistical analyses were performed. Two-way ANOVA with repeated measures [condition (BFR-Tr, MH-Tr, Ctrl) by time (pre, post)] was used to evaluate the training effects for all dependent variables. When significant main effects and/or interaction were observed, post hoc testing was performed using Tukey post hoc test. Statistical significance was set at $p<0.05$. The sample size was estimated from a priori power analysis [45] to detect differences (power of 0.80 an $\alpha$ of 0.05 , two-tailed, and an effect size of 1.6-2.1) in knee extension and leg press 1RM ( $n=6$, female) for the interventions planned by reference to the result of previous BFR study [21]. Consequently, it was determined that a minimum of 5-8 BFR training and 5-8 control participants were required to test both the main and interaction effects. Pre/ Post effect sizes (ESs, Cohen's $d$ ) for muscle CSA, MVIC and $1 \mathrm{RM}$ were calculated using the following formula: [post mean - pre mean]/pre SD; $d<0.2$ is a trivial effect, $d$ $=0.2-0.5$ is a small effect, $d=0.5-0.8$ is a moderate effect, and $d>0.8$ is a large effect [45].

\section{ACKNOWLEDGMENTS}

The authors thank the participants who participated in this study. We also thank Dr. Ken Masamune (Tokyo Women's Medical University), Dr. Tatsuya Yamasoba, Dr. Haruko Iida, Ms. Hitomi Koshi, Mr. Junxiao Nie, and Mr. Hiroyuki Imuta (University of Tokyo) and for helpful discussion and technical support.

\section{CONFLICTS OF INTEREST}

Our department (University of Tokyo) was funded by KAATSU Japan Co., Ltd. This study used equipment manufactured by the KAATSU Japan Co., Ltd.

\section{GRANT SUPPORT}

This study was supported, in part, by Grant-in-aid (\#24300189 to TN and \#25750288 to TY) from the Japan Ministry of Education, Culture, Sports, Science, and Technology, the Nakatomi Foundation (to TY) and the Vehicle Racing Commemorative Foundation (to TN).

\section{REFERENCES}

1. Haykowsky MJ, Brubaker PH, Morgan TM, Kritchevsky S, Eggebeen J, Kitzman DW. Impaired aerobic capacity and physical functional performance in older heart failure patients with preserved ejection fraction: role of lean body mass. J Gerontol A Biol Sci Med. Sci. 2013; 68: 968-975.

2. Visser M, Goddpaster BH, Kritchevsky SB, Newman AB, Nevitt M, Rubin SM, Simonsick EM, Harris TB. Muscle mass, muscle strength, and muscle fat infiltration as predictors of incident mobility limitations in wellfunctioning older persons. J Gerontol A Biol Sci Med. Sci. 2005; 60: 324-333.

3. American College of Sports Medicine. American College of Sports Medicine position stand. Progression models in resistance training for healthy adults. Med Sci Sports Exerc 2009; 41: 687-708.

4. Fiatarone MA, Marks EC, Ryan ND, Meredith CN, Lipsitz LA, Evans WJ. High-intensity strength training in nonagenarians. Effects on skeletal muscle. JAMA. 1990; 263: 3029-3034.

5. Frontera WR, Meredith CN, O_Reilly KP, Knuttgen $\mathrm{HG}$, Evans WJ. Strength conditioning in older men: skeletal muscle hypertrophy and improved function. J Appl Physiol. 1988; 64: 1038-1044.

6. Aagaard P, Suetta C, Caserotti P, Magnusson SP, Kjaer $\mathrm{M}$. Role of the nervous system in sarcopenia and muscle atrophy with aging: strength training as a countermeasure. Scand J Med Sci. Sports 2010; 20: 49-64.

7. Ribeiro F, Teixeira F, Brochado G, Oliveira J. Impact of low cost strength training of dorsi- and plantar flexors on balance and functional mobility in institutionalized elderly people. Geriatr Gerontol Int. 2009; 9: 75-80.

8. Zion AS, De Meersman R, Diamond BE, Bloomfield DM. A home-based resistance-training program using elastic bands for elderly patients with orthostatic hypotension. Clin Auton Res. 2003; 13: 286-292.

9. Mikesky A, Topp R, Wigglesworth J, Harsha DM, Edwards J. Efficacy of a home based training program for older adults using elastic tubing. Eur J Appl Physiol Occup Physiol. 1994; 69: 316-320.

10. Colado JC, Triplett NT. Effects of a short-term resistance program using elastic bands vs. weight machines for sedentary middle-aged women. J Strength Cond Res. 2008; 22: $1441-1448$.

11. Takarada Y, Nakamura Y, Aruga S, Onda T, Miyazaki S, Ishii N. Rapid increase in plasma growth hormone after low-intensity resistance exercise with vascular occlusion. J Appl Physiol. 2000; 88: 61-65.

12. Abe T, Yasuda T, Midorikawa T, Sato Y, Kearns CF, Inoue K, Koizumi K, Ishii N. Skeletal muscle size and circulating IGF-1 are increased after two weeks of twice daily "KAATSU" resistance training. Int. J. Kaatsu Training Res. 2005; 1: 6-12.

13. O'Rourke, M. Arterial stiffness, systolic blood pressure, and logical treatment of arterial hypertension. Hypertension. 1990; 15: 339-347.

14. Monahan KD, Tanaka H, Dinenno FA, Seals DR. Central arterial compliance is associated with age- and habitual exercise-related differences in cardiovagal baroreflex 
sensitivity. Circulation 2001; 104: 1627-1632.

15. Miyachi M, Donato AJ, Yamamoto K, Takahashi K, Gates PE, Moreau KL, Tanaka H.. Greater age-related reductions in central arterial compliance in resistance-trained men. Hypertension. 2003; 41: 130-135.

16. Miyachi M, Kawano H, Sugawara J, Takahashi K, Hayashi K, Yamazaki K, Tabata I, Tanaka H. Unfavorable effects of resistance training on central arterial compliance: a randomized intervention study. Circulation. 2004; 110: 2858-2863.

17. Casey DP, Pierce GL, Howe KS, Mering MC, Braith RW. Effect of resistance training on arterial wave reflection and brachial artery reactivity in normotensive postmenopausal women. Eur J Appl Physiol. 2007; 100: 403-438.

18. Rakobowchuk M, McGowan CL, de Groot PC, Bruinsma D, Hartman JW, Phillips SM, MacDonald MJ. Effect of whole body resistance training on arterial compliance in young men. Exp Physiol. 2005; 90: 645-651.

19. Fahs CA, Rossow LM, Thiebaud RS, Loenneke JP, Kim D, Abe T, Beck TW, Feeback DL, Bemben DA, Bemben MG. Vascular adaptations to low-load resistance training with and without blood flow restriction. Eur J Appl Physiol. 2014; 114: 715-724.

20. Ozaki H, Yasuda T, Ogasawara R, Sakamaki-Sunaga M, Naito H, Abe T. Effects of high-intensity and blood flowrestricted low-intensity resistance training on carotid arterial compliance: role of blood pressure during training sessions. Eur J Appl Physiol. 2013; 113: 167-174.

21. Yasuda T, Fukumura K, Fukuda T, Uchida Y, Iida H, Meguro M, Sato Y, Yamasoba T, Nakajima T. Muscle size and arterial stiffness after blood flow-restricted lowintensity resistance training in older adults. Scand J Med Sci Sports. 2014; 24: 799-806.

22. Miyatani M, Kanehisa H, Azuma K, Kuno S, Fukunaga T. Site-related differences in muscle loss with aging: a cross sectional survey on the muscle thickness in Japanese men and Age-related muscle loss in Japanese adults 150 women aged 20 to 79 years. Int J Sport Health Sci. 2003; 1: 34-40.

23. Abe T, Sakamaki M, Yasuda T, Bemben MG, Kondo M, Kawakami Y, Fukunaga T. Age related, site-specific muscle loss in 1507 Japanese men and women aged 20 to 95 years. Journal of Sports Science and Medicine. 2011; 10: 145-150.

24. Thiebaud RS, Loenneke JP, Fahs CA, Rossow LM, Kim D, Abe T, Anderson MA, Young KC, Bemben DA, Bemben MG. The effects of elastic band resistance training combined with blood flow restriction on strength, total bone-free lean body mass and muscle thickness in postmenopausal women. Clin Physiol Funct Imaging. 2013; 33: 344-352.

25. Petrella JK, Kim JS, Mayhew DL, Cross JM, Bamman MM. Potent myofiber hypertrophy during resistance training in humans is associated with satellite cell-mediated myonuclear addition: a cluster analysis. J Appl Physiol. 2008; 104: 1736-1742.
26. Nielsen JL, Aagaard P, Bech RD, Nygaard T, Hvid LG, Wernbom M, Suetta C, Frandsen U. Proliferation of myogenic stem cells in human skeletal muscle in response to low-load resistance training with blood flow restriction. J Physiol. 2012; 590: 4351-4361.

27. Loenneke JP, Fahs CA, Rossow LM, Abe T, Bemben MG. The anabolic benefits of venous blood flow restriction training may be induced by muscle cell swelling. Med Hypotheses. 2012; 78: 151-154.

28. Yasuda T, Loenneke JP, Thiebaud RS, Abe T. Effects of low-intensity blood flow restricted concentric or eccentric training on muscle size and strength. PLoS One. 2012; 12 : e52843.

29. Yasuda T, Abe T, Brechue WF, Iida H, Takano H, Meguro K, Kurano M, Fujita S, Nakajima T. Venous blood gas and metabolite response to low-intensity muscle contractions with external limb compression. Metabolism. 2010; 59: 1510-1519.

30. Keller U, Szinnai G, Bilz S, Berneis K. Effects of changes in hydration on protein, glucose and lipid metabolism in man: impact on health. Eur J Clin Nutr. 2003; 57(Suppl 2): S69-S74.

31. Millar ID, Barber MC, Lomax MA, Travers MT, Shennan DB. Mammary protein synthesis is acutely regulated by the cellular hydration state. Biochem Biophys Res Commun. 1997; 230: 351-355.

32. Nigam A, Mitchell GF, Lambert J, Tardif JC. Relation between conduit vessel stiffness (assessed by tonometry) and endothelial function (assessed by flow-mediated dilatation) in patients with and without coronary heart disease. Am J Cardiol. 2003; 92: 395-399.

33. Clark BC, Manini TM, Hoffman RL, Williams PS, Guiler MK, Knutson MJ, McGlynn ML, Kushnick MR. Relative safety of 4 weeks of blood flow-restricted resistance exercise in young, healthy adults. Scand J Med Sci Sports. 2011; 21: 653-662.

34. Nakajima T, Kurano M, Iida H, Takano H, Oonuma H, Morita T, Meguro K, Sato Y, Nagata T, KATTSU Training Group. Use and safety of KAATSU training: Results of a national survey. Int. J. KAATSU Training Res. 2006; 2: 5-13.

35. Lagally KM, Robertson RJ. Construct validity of the OMNI resistance exercise scale. J Strength Cond Res. 2006; 20 : 252-256.

36. Greig CA, Young A, Skelton DA, Pippet E, Butler FM, Mahmud SM. Exercise studies with elderly volunteers. Age Ageing. 1994; 23: 185-189.

37. The Japanese Society of Hypertension Guidelines for Management of Hypertension (JSH 2014). Hypertens Res. 2014; 37: 253-392.

38. Page P, Labbe A, Topp RV. Clinical force production of thera-band elastic bands. J Orthop Sports Phys Ther. 2000; 30: A47. 
39. Yasuda T, Ogasawara R, Sakamaki M, Ozaki H, Sato $\mathrm{Y}$, Abe T. Combined effects of low-intensity blood flow restriction training and high-intensity resistance training on muscle strength and size. Eur J Appl Physiol. 2011; 111: 2525-2533.

40. Ozaki H, Sakamaki M, Yasuda T, Fujita S, Ogasawara R, Sugaya M, Nakajima T, Abe T. Increases in thigh muscle volume and strength by walk training with leg blood flow reduction in older participants. J Gerontol A Biol Sci Med Sci. 2010; 66: 257-263.

41. Wathen D. Load Assignment. In: Baechle TR, editor. Essentials of Strength Training and Conditioning. Champaign, IL: Human Kinetics, 1994: 435-439.

42. Takazawa K, Kobayashi H, Shindo N, Tanaka N, Yamashina A. Relationship between radial and central arterial pulse wave and evaluation of central aortic pressure using the radial arterial pulse wave. Hypertens Res. 2007; 30: 219-228.
43. Takase H, Dohi Y, Kimura G. Distribution of central blood pressure values estimated by Omron HEM-9000AI in the Japanese general population. Hypertens Res. 2013; 36: 5057.

44. Shirai K, Otsuka K, Takata M. A novel blood pressure independent arterial wall stiffness parameter: cardio-ankle vascular index (CAVI). J Atheroscler Thromb. 2006; 13: 101-107.

45. Cohen J. Statistical power analysis for the behavioral sciences. 2nd Ed. Hillsdale, NJ: Lawrence Erlbaum Associates Inc. 1988; p.19-39. 